\title{
Improvements of Webometrics by Using Sentiment Analysis for Better Accessibility of the Web
}

\author{
Radek Malinský and Ivan Jelínek \\ Department of Computer Science and Engineering, Faculty of Electrical Engineering \\ Czech Technical University in Prague, \\ Karlovo náměstí 13, 12135 Prague, Czech republic \\ \{malinrad, jelinek\}@fel.cvut.cz \\ http://webing.felk.cvut.cz
}

\begin{abstract}
The paper discusses the webometric model for effective acquirement of relevant information from the web that would better separate the useful data from the useless. Our research emphasis has been placed on techniques that would better reflect the semantic content of single pages. Webometrics is purely a quantitative approach to the web, which can be enhanced by qualitative methods and thereby allows us to expand the possibilities of a study problem. Sentiment analysis may be used as a qualitative complement to quantitative approach. This analysis provides a technique of sophisticated analysis of sentences using mathematical and statistical methods and linguistic analysis of text. Extension of the webometric techniques of sentiment analysis methods leads up to a better machine understanding of a web page and its overall semantic meaning. It can be assumed that the designed model will reduce the irrelevant web search results and thereby facilitate user access to the information on the web. The introductory part of the paper explains the concept of the sentiment analysis and the basic functional background of the webometric techniques.
\end{abstract}

Keywords: Webometrics, Sentiment analysis, Semantics, Web 2.0.

\section{Introduction}

Until recently, webometric research has been focused on the evaluation of pages without any previous knowledge. Many of these quantitative studies have focused on hyperlinks. For example, research focused on the issue, whether interlinking between local government bodies in Finland follows a strong geographic, or rather a geopolitical pattern [3], or a correlation between universities' research and their presence on the web exists [1. The quality of pages is determined by bibliometric and informetric approaches [4, [9]. These approaches are very simple and do not reflect the semantic content of single pages.

The volume of information on the web has been growing at a very high rate and becoming a network of heterogeneous data, this makes things difficult to

F. Daniel and F.M. Facca (Eds.): ICWE 2010 Workshops, LNCS 6385, pp. 581 586, 2010.

(C) Springer-Verlag Berlin Heidelberg 2010 
find and is therefore not almost useful. It is necessary to design suitable metric for such volume of information, which would reflect semantic content of pages in the better way. However, the primary task is to define criteria for the quality assessment of found information, and "distance" between relevant information. On this basis, we can design rules for evaluation of semantic content in relation to user's queries.

One of the options for more accurate comprehension of semantic information is to use a sophisticated analysis of sentences using mathematical and statistical methods and linguistic analysis of text (called Sentiment Analysis [6]).

The knowledge gained will be useful for algorithm design to facilitate user access to the information on the web, and to obtain the public opinion on specific issues above all. The algorithm will be based on current webometric techniques [1], 2], 4], 10, 11] extended by sentiment analysis [6], 7], 8].

\section{Webometrics}

Webometrics is a scientific discipline that studies the quantitative aspects of information resources and their use. Webometrics is based on informetric and bibliometric methods 9 . The information sources that are studied by webometrics are web documents. According to narrow definition, webometrics encompass two basic categories: web link structure analysis and the evaluation of search engines using informetric methods.

Current search engines are based on the idea of citation analysis and impact factor 4], 9]. This strategy especially excels in queries to a specific case, but when you enter a complex query, it returns a large number of irrelevant links and does not reflect the semantic content of single pages.

Webometrics is used in scientific communication, especially for citation counts (scientometrics) and for analysis of electronic journals and universities websites [5], 9. Non-academic parts of the web are rather heterogeneous, therefore; it is difficult, but not impossible to find common webometric characteristics.

In recent years, there have been efforts to apply webometric techniques to nonacademic parts of the web. Google PageRank 4 or Ranking of World Universities [1] are good examples of successful projects. Webometrics in the area of Web 2.01 has been mainly used to find information on blogs [2] and for trend detection [10]. In the beginning, it was a simple context analysis based on counting how often searched word was mentioned online. Current research is focused on sophisticated analysis of sentences [7, 8], which aims to determine the polarity of the text and the attitude of a writer with respect to some topic.

Several types of techniques have been designed during the gradual development of webometrics: web link structure analysis, web page content analysis, web usage analysis, web technology analysis, etc. The first two mentioned techniques are the most important for our research at this point.

${ }^{1}$ The term "Web 2.0" is a designation for the developmental stage of the web, where static content is replaced by space for creating and sharing content. 


\subsection{Link Analysis}

Link Analysis [1], [3], [5] is used to determine the impact of information, documents, pages or organizations on the web, or to determine the online relationships between them. Input data is gathered either from commercial search engines or from research crawlers. The evaluated output can be reported as inlink counts or as a network diagram with nodes representing web sites and arrows between the nodes representing the links between them.

PageRank 4 used by the Google internet search engine is the most reliable and effective link analysis algorithm. The algorithm evaluates the relevance of search result pages by the number of inlinks and by the quality of source of these inlinks. The quality of inlinks has a much higher significance than the number of inlinks.

\subsection{Web Mention Analysis}

Web Mention Analysis [1] is the evaluation of the "web impact" of documents or ideas by counting how often they are mentioned online. Assessment is a combination of several types of methods:

Web Mentions. This method determines the popularity estimation of ideas or documents using reported hit count estimates from commercial search engines. The hit count estimates are the numbers reported by search engines in their result pages as the estimated maximum number of matching pages.

Content Analysis. Content Analysis is a systematic separation into categories, such as the document type, national origins, industrial sector, etc. It is used to reduce the irrelevant search results that have nothing to do with the specific category.

Hyperlink Analysis. Hyperlink Analysis is based on the extraction of information from URLs. This is very useful in Content Analysis because URL extraction can provide information such as the geographic spread or the type of organization that is interested in the document.

\section{Sentiment Analysis}

Sentiment Analysis or Opinion Mining enables us to automatically detect opinions from structured but also unstructured data. This involves several research areas such as natural language processing, computational linguistic and text mining. The research in this field originated from the demand of commercial companies, who wanted to know public opinion on price, quality and other features of their products [6].

Before the massive spread of the Internet, companies had been gaining customer feedback via phone, email surveys or interviews. It was very slow, expensive and annoying for some customers. With the Internet, companies may be 
able to gain feedback from comments in e-shops, blogs, customer reviews, social networks, etc. These methods of obtaining information are very fast and thanks to that the company that has just launched a major advertising campaign may gain quick public feedback on its impact.

The main goal of sentiment analysis is to identify positive/negative polarity of the text and recognize a subjective/objective impression of the text [8]. As a sub task, analysis is capable to determine the attitude of a writer with respect to some topic. The attitude may express affective state of the author when writing or intended emotional effect which author wishes to present to the reader.

\section{Webometrics + Sentiment Analysis}

Current research on Sentiment Analysis has made great strides and is currently used in many industrial applications, such as the analysis of the comments of goods in e-shops [6] or the videos on YouTube [7]. We built our research on the achievements in this area [6], 7], 8, , 12, which we want to use for improvements of webometrics for better accessibility of the web. Webometrics is purely a quantitative approach to the web, which can be enhanced by qualitative methods and thereby allows us to expand the possibilities of a study problem [11.

Original webometric techniques [1], 2], 4], 10, 11] improved searching and provided trend detection. However, they are not able to distinguish a polarity of the text, its semantic meaning and the emotional state of the author. Extension of the webometric techniques of sentiment analysis methods leads up to gaining insights into a public opinion with respect to some topic and to a better machine understanding of the text. Better understanding of the content on the web site could have a significant impact on the quality of site evaluation.

The aim of our work is to propose a theoretical model for a more effective way of obtaining relevant information from the web. The model builds on webometrics and starts from the idea that almost any text can be machine-recognized. This idea is supported by current research in sentiment analysis [6], [7], [8], which aims at sophisticated analysis of sentences using mathematical and statistical methods and linguistic analysis of text.

The model provides complete content analysis of crawled pages. The analysis is performed in three phases. The first phase; Webometric Web Mention Analysis, determines some basic information about the document such as the document type, geographic spread, type of organization that is interested in the document etc. and chooses keywords or entire phrases which represent it. The second phase; Sentiment Analysis, determines the polarity and impression of the text and evaluates the selected key words and phrases. The third phase; Webometric Link Analysis, determines the impact of an analyzed document. The next part of the model is a proper citation index that stores the output of the content analysis and allows quick and easy searches with regard to users' queries.

Web 2.0 can serve as a data source for our research because it contains vast amount of information from many different users. Thanks to blogs and social networks; its popularity has been constantly rising and the volume of information has been growing at a very high rate. 


\section{Work Plan}

Our research should be improving the content representation of a web page and its semantic meaning. There are several important steps leading to the completion of our work. 1. Design an appropriate assessing method of analyzed information that would better separate the useful data from the useless. 2. Propose an appropriate citation index structure and algorithm for the information obtained from it. 3. Create and implement an experimental system for gathering and processing data from Web 2.0, which will be able to verify the theoretical assumptions.

Furthermore, the final model will be used to develop an algorithm which improves the quality of search engines on the principle of webometrics. This will lead to a better machine understanding of user queries and thereby the reduction of irrelevant web search results.

Acknowledgments. This research has been supported by MSMT under research program No. 6840770014. This research has been supported by the Grant Agency of the CTU in Prague, grant No. SGS10/202/OHK3/2T/13.

\section{References}

1. Aguillo, I.F., Ortega, J.L., Fernández, M.: Webometric Ranking of World Universities: Introduction, Methodology, and Future Developments. Higher Education in Europe 33(3), 234-244 (2008)

2. Han, S.K., Shin, D., Jung, J.Y., Park, J.: Exploring the Relationship between Keywords and Feed Elements in Blog Post Search. World Wide Web - Internet and Web Information Systems 12(4), 381-398 (2009)

3. Holmberg, K., Thelwall, M.: Local government web sites in Finland: A geographic and webometric analysis. Scientometrics 79(1), 157-169 (2009)

4. Langville, A.N., Meyer, C.D.: Google's PageRank and Beyond: The Science of Search Engine Rankings. Princeton University Press, Princeton (2006), ISBN 9780691122021

5. Ortega, J., Aquillo, I.F.: Visualization of the Nordic academic web: Link analysis using social network tools. Information Processing \& Management 44(4), 1624 1633 (2008)

6. Pang, L., Lee, L.: Opinion mining and sentiment analysis. Foundations and Trends in Information Retrieval 2(1), 1-135 (2008)

7. Potthast, M., Becker, S.: Opinion Summarization of Web Comments. In: Gurrin, C., He, Y., Kazai, G., Kruschwitz, U., Little, S., Roelleke, T., Rüger, S., van Rijsbergen, K. (eds.) ECIR 2010. LNCS, vol. 5993, pp. 668-669. Springer, Heidelberg (2010)

8. Prabowo, R., Thelwall, M.: Sentiment analysis: A combined approach. Journal of Informetrics 3(1), 143-157 (2009)

9. Thelwall, M.: Bibliometrics to Webometrics. Journal of Information Science 34(4), 605-621 (2008) 
10. Thelwall, M.: Blog searching: The first general-purpose source of retrospective public opinion in social science? Online Information Review 31(3), 277-289 (2007)

11. Thelwall, M.: Introduction to Webometrics: Quantitative Web Research for the Social Sciences. Morgan \& Claypool, San Rafael (2009), ISBN 9781598299946

12. Thelwall, M., Wilkinson, D.: Public dialogs in social network sites: What is their purpose? Journal of the American Society for Information Science and Technology $61(2), 392-404(2010)$ 